\title{
OPTIMAL PARISIAN-TYPE DIVIDEND PAYMENTS PENALIZED BY THE NUMBER OF CLAIMS FOR THE CLASSICAL AND PERTURBED CLASSICAL RISK PROCESS
}

BY

\author{
IRMINA CZARNA* (WROCŁAW), YANHONG LI (ChENGDU), \\ ZBIGNIEW PALMOWS K I** (Wroceaw), AND CHUNMING ZHAO*** (Chengdu)
}

\begin{abstract}
We consider the classical risk process (the case $\sigma=0$ ) and the classical risk process perturbed by a Brownian motion (the case $\sigma>0$ ). We analyze the expected NPV describing the mean of the cumulative discounted dividend payments paid up to the Parisian or classical ruin time and further penalized by the number of claims that appeared up to that time. We identify this function for a constant barrier strategy and we find sufficient conditions for this strategy to be optimal. We also analyze a numerical example of exponential claim sizes.
\end{abstract}

2020 Mathematics Subject Classification: Primary 60J99; Secondary 93E20, 60G51.

Key words and phrases: classical risk model, diffusion process, number of claims, Parisian ruin, dividend.

\section{INTRODUCTION}

The classical optimal dividend problem has been been considered by many authors since de Finetti [9] who introduced it to address the objection that the risk process has the unrealistic property that it converges to infinity with probability 1. Gerber and Shiu [13], Asmussen and Taksar [1] and Jeanblanc and Shiryaev [14] considered the optimal dividend problem in the Brownian motion setting. Azcue and Muler [4] and Schmidli [29] studied the optimal dividend strategy under the Cramér-Lundberg model using a Hamilton-Jacobi-Bellman (HJB) system of equations. Further, Avram et al. [2], [3], Kyprianou and Palmowski [18], Loeffen

* Supported by National Science Centre Grant No. 2015/19/D/ST1/01182.

${ }^{* *}$ Supported by National Science Centre Grant No. 2013/09/B/HS4/01496.

*** Supported by the Fundamental Research Funds for the Central Universities (Grant No. 2682017CX065). 
[22], [23], Loeffen and Renaud [25], Czarna and Palmowski [7] and many other authors analyzed the dividend problem for the Lévy risk process using the probabilistic approach.

In this paper we consider a classical risk process perturbed by a Brownian motion. Such a process is a particular example of a Lévy risk process and it covers a vast range of examples which are of interest for insurance companies. The classical risk process models so-called 'large' claims and an independent Brownian motion models 'small' claims.

Our approach is to combine two new ingredients appearing in the expected NPV. The first one concerns choosing the Parisian ruin time instead of the classical ruin time. Parisian ruin occurs if the risk process stays below zero for a continuous time interval of length greater than a fixed $d \geqslant 0$. The name comes from the Parisian option which is activated or canceled depending on whether the underlying asset price stays above or below the barrier over an uninterrupted period (see Chesney et al. [5] and Dassios and $\mathrm{Wu}[8]$ ). We believe that allowing Parisian delay could be reasonable in many situations, as it gives the insurance companies a chance to achieve solvency. Still, the particular case $d=0$ brings us to the classical set-up, which is also considered in this paper. The second new factor $(r \in(0,1])$ in the value function concerns an additional component related to the total number of claims which arrived up to Parisian ruin. Taking into account this extra factor based on the total number of claims/losses that arrived up to the ruin time allows one to diminish (penalize) the objective value in the case of a large number of claims and increase it in the case of a small number of claims. This is a very natural and practical feature of dividend payments. A similar penalization, based on the number of reported claims only, can be observed in widely-used bonusmalus system. There is another argument for choosing the penalization based on the number of claims. As noted by Gerber [11], de Finetti's criterion with $r=1$ (hence not taking into account the number of claims) has a major disadvantage: it does not directly protect the company and it maximizes the gain of the beneficiaries of the dividend payments only. As a consequence, it generates optimal strategies that are hardly acceptable from the practical point of view. Considering the case $r<1$ makes this criterion more realistic, since it additionally penalizes the event of a large number of claims that the company wants to avoid. Therefore we believe that the analysis presented in this paper is of value for actuarial applications.

In Theorem 3.5 we identify the function described above for a barrier strategy $\left(\pi_{a}\right)$ according to which all the surplus above a fixed level $a$ is paid as dividends. We also find sufficient conditions for this strategy to be optimal. Finally, we calculate the value function for a process with exponentially distributed claims.

The paper is organized as follows. In Section 2 we introduce the basic notions, notations and describe the model. In Section 3 we analyze in detail the barrier strategy $\pi_{a}$ and the form of the expected NPV. In Section 4 we find sufficient conditions for the barrier strategy to be optimal. In the last section we present an 
example with exponentially distributed claims.

\section{PROBLEM FORMULATION}

We consider the following surplus process in continuous time:

$$
X_{t}=x+c t-\sum_{i=1}^{N_{t}} C_{i}+\sigma B_{t},
$$

where the non-negative constant $x$ denotes the initial reserve (later we underline this initial capital by adding it as a subscript to probability measures, $\mathbb{P}_{x}(\cdot)=$ $\mathbb{P}\left(\cdot \mid X_{0}=x\right)$ with $\mathbb{P}:=\mathbb{P}_{0}$, and to the corresponding expectations, $\mathbb{E}_{x}$ with $\left.\mathbb{E}:=\mathbb{E}_{0}\right)$. The arrival process is a homogeneous Poisson process $N_{t}$ with intensity $\lambda$, describing the number of claims that appeared up to time $t$. The random variables $\left\{C_{i}\right\}_{i=1}^{\infty}$ are claim sizes which are independent, identically distributed (i.i.d.), non-negative and also independent of $N_{t}$. We denote by $f(x)$ and $F(x)$ the density and the distribution function of the claims, respectively. Throughout this paper we assume that the claim density $f$ is continuously differentiable. The positive constant $c=\lambda \mathbb{E}\left(C_{1}\right)(1+\theta)$ is the rate of premium income and $\theta>0$ is the relative security loading factor. The process $\left\{B_{t}\right\}_{t \geqslant 0}$ is a standard Wiener process that is independent of the aggregate claims process $\sum_{i=1}^{N_{t}} C_{i}$, and $\sigma \geqslant 0$ is a dispersion parameter (see Figure 1 for an example of a sample path where $\sigma=0$ ).

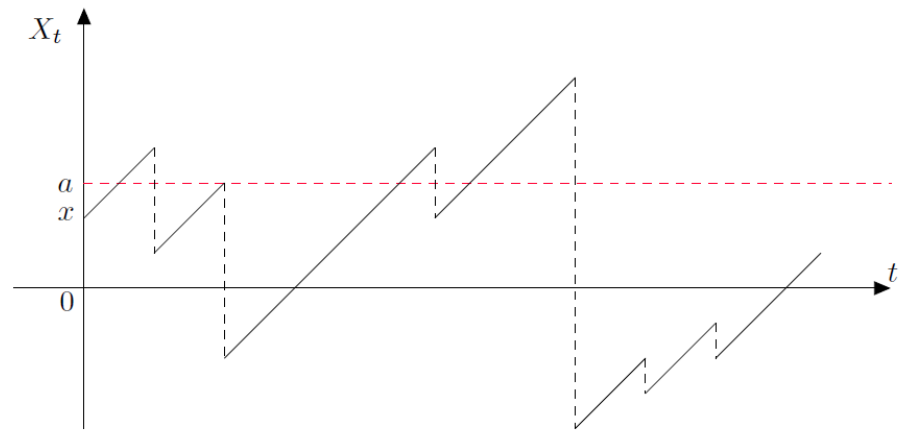

FiguRE 1. A sample path of the original surplus process $X_{t}$.

We denote a dividend (control strategy) by $\pi$, where $\pi=\left\{L_{t}^{\pi}: t \geqslant 0\right\}$ is a non-decreasing left-continuous adapted process which starts at zero. The random variable $L_{t}^{\pi}$ represents the cumulative dividends the company has paid out up to time $t$ under the control strategy $\pi$. We denote by

$$
U_{t}^{\pi}=X_{t}-L_{t}^{\pi}
$$

the controlled risk process under the dividend strategy $\pi$. For fixed $d \geqslant 0$ let

$$
\tau^{\pi, d}:=\inf \left\{t>0: t-\sup \left\{s<t: U_{s}^{\pi} \geqslant 0\right\}>d, U_{t}^{\pi}<0\right\}
$$




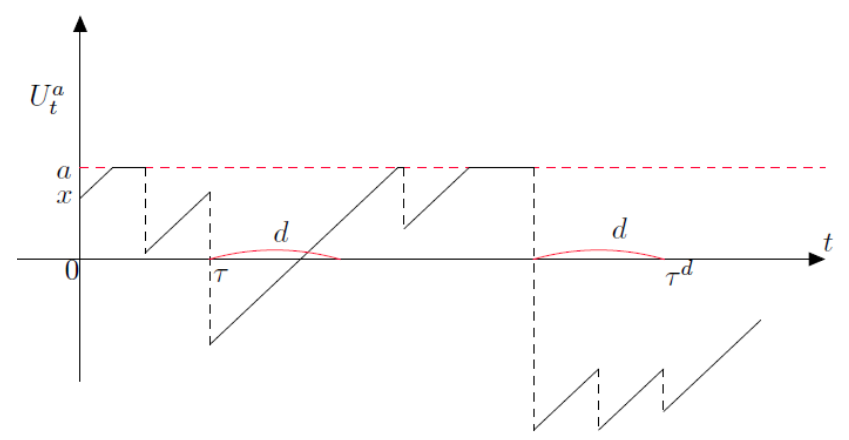

Figure 2. A sample path of the regulated surplus process $U_{t}^{a}$ (case $\sigma=0$ ).

be the Parisian ruin time, that is, the ruin occurs if the regulated process $U_{t}^{\pi}$ stays below zero for a continuous time interval of length greater than $d$ (see Figure 2). The case $d=0$ corresponds to the classical ruin time:

$$
\tau^{\pi, 0}:=\inf \left\{t \geqslant 0: U_{t}^{\pi}<0\right\} .
$$

Now for any $d \geqslant 0$ we formally define the expected NPV for a dividend strategy $\pi$ as follows:

$$
v^{\pi}(x):=\mathbb{E}_{x}\left[r^{N_{\tau} \pi, d} \int_{0}^{\tau^{\pi, d}} e^{-q t} d L_{t}^{\pi}\right]
$$

where $r \in(0,1]$ is a constant and $q>0$ is a discounting rate. By the definition above, it follows that for $d=0$,

$$
\begin{array}{ll}
v^{\pi}(x)=0 & \text { if } x<0 \text { and } \sigma=0, \\
v^{\pi}(x)=0 & \text { if } x \leqslant 0 \text { and } \sigma>0,
\end{array}
$$

and for $d>0$,

$$
\begin{array}{ll}
v^{\pi}(x)=0 & \text { if } x<-c d \text { and } \sigma=0, \\
\lim _{x \rightarrow-\infty} v^{\pi}(x)=0 & \text { if } \sigma>0,
\end{array}
$$

since the ruin (classical or Parisian) will occur before we collect any dividends. A strategy $\pi$ is called admissible if ruin does not occur by a dividend payout, that is, $L_{t+}^{\pi}-L_{t}^{\pi} \leqslant U_{t}^{\pi}$ for $t<\tau^{\pi}$. Let $\Pi$ denote the set of all admissible dividend strategies. The objective of the beneficiaries of an insurance company is to maximize $v^{\pi}(x)$ over all admissible strategies $\pi$, that is, to find the optimal value function $v^{*}$ given by

$$
v^{*}(x)=\sup _{\pi \in \Pi} v^{\pi}(x)
$$

and to identify the optimal strategy $\pi^{*} \in \Pi$ such that

$$
v^{\pi^{*}}(x)=v^{*}(x) .
$$




\section{BARRIER STRATEGY $\pi_{a}$}

The crucial dividend distribution policy is the barrier policy $\pi_{a}=\left\{L_{t}^{a}:=L_{t}^{\pi_{a}}\right.$ : $t \geqslant 0\}$ of transferring all surpluses above a given level $a$ as dividends (see Figure 2). In this case $L_{t}^{a}:=\left(a \vee \sup _{s \leqslant t} X_{s}\right)-a$. For this specific strategy we denote

$$
U_{t}^{a}:=U_{t}^{\pi_{a}}=X_{t}-L_{t}^{a}, \quad \tau^{d}:=\tau^{\pi_{a}, d},
$$

with $\tau:=\tau^{0}$ being the classical ruin time, and

$$
v^{a, d}(x):=v^{\pi_{a}, d}(x)=\mathbb{E}_{x}\left[r^{N_{\tau^{d}}} \int_{0}^{\tau^{d}} e^{-q t} d L_{t}^{a}\right]
$$

In this paper we focus on finding the function $v^{a, d}(x)$. Later we will identify the optimal barrier $a^{*}$ maximizing $v^{a, d}(x)$. Finally, we show that in most of the known cases of the claim size density $f$ the strategy $\pi_{a^{*}}$ is indeed optimal, that is,

$$
v^{*}(x)=v^{a^{*}, d}(x) .
$$

3.1. The form of the expected NPV. We start by identifying the function $v^{a, d}$ under the barrier strategy $\pi_{a}$ for a fixed $a$. Moreover in the following lemma we present the basic properties of the function $v^{a, d}$.

LEMMA 3.1. The function $v^{a, d}$ is twice continuously differentiable for all $x<a$ and solves the differential equation

$$
\frac{\sigma^{2}}{2}\left(v^{a, d}\right)^{\prime \prime}(x)+c\left(v^{a, d}\right)^{\prime}(x)-(\lambda+q) v^{a, d}(x)+\lambda r \int_{0}^{\infty} v^{a, d}(x-y) f(y) d y=0 .
$$

Proof. The proof of the twice continuous differentiability is based on Li [19, Thms. 2 and 3], so we skip all the details. Still our reasoning requires some modifications. The main one is that we add a new term $I_{4}(x)$, which is given by

$$
\begin{aligned}
I_{4}(x)= & \int_{0}^{t_{0}} \lambda r e^{-(\lambda+q) s} d s \int_{-b}^{b} H(b, s, y) d y \\
& \times \int_{x+c s+\sigma y}^{\infty} \mathbb{E}_{x+c s+\sigma y-z}\left[r^{N} \tau_{0}^{+} e^{-q \tau_{0}^{+}}, \tau_{0}^{+}<d\right] v^{a, d}(0) f(z) d z \\
= & \int_{0}^{t_{0}} \lambda r e^{-(\lambda+q) s} d s \int_{-b}^{b} H(b, s, y) d y \\
& \times \int_{x+c s+\sigma y}^{\infty} \phi(x+c s+\sigma y-z, d) v^{a, d}(0) f(z) d z,
\end{aligned}
$$


where for $u \geqslant 0$,

$$
\phi(-u, d):=\mathbb{E}_{-u}\left[r^{N_{\tau_{0}^{+}}} e^{-q \tau_{0}^{+}}, \tau_{0}^{+}<d\right]=\mathbb{E}\left[r^{N_{\tau_{u}^{+}}} e^{-q \tau_{u}^{+}}, \tau_{u}^{+}<d\right]
$$

$0 \leqslant t_{0} \leqslant(a-x) /(2 c), \tau_{0}^{+}$denotes the first time that $X_{t}$ up-crosses zero, and $H(b, s, y)=\mathbb{P}\left(B_{s} \in d y, \tau_{b}>s\right)$ for $\tau_{b}=\inf \left\{s:\left|B_{s}\right|=b\right\}$. Indeed, if we take $\tau:=t_{0} \wedge \tau_{b} \wedge T_{1}$, where $T_{1}$ is the occurrence time of the first claim, which is exponentially distributed with parameter $\lambda$, then from the strong Markov property we get

$$
\begin{aligned}
v^{a, d}(x)= & \mathbb{E}_{x}\left[r^{N_{\tau}} e^{-q \tau} v^{a, d}\left(U_{\tau}^{a}\right)\right] \\
= & e^{-q t_{0}} \mathbb{E}_{x}\left[v^{a, d}\left(x+c t_{0}+\sigma B_{t_{0}}\right) \mathbf{1}_{\left\{t_{0}<\tau_{b} \wedge T_{1}\right\}}\right] \\
& +\mathbb{E}_{x}\left[e^{-q \tau_{b}} v^{a, d}\left(x+c \tau_{b}+\sigma B_{\tau_{b}}\right) \mathbf{1}_{\left\{\tau_{b} \leqslant t_{0} \wedge T_{1}\right\}}\right] \\
& +\mathbb{E}_{x}\left[e^{-q T_{1}} r^{N_{T_{1}}} v^{a, d}\left(x+c T_{1}+\sigma B_{T_{1}}-C_{1}\right) \mathbf{1}_{\left\{T_{1}<\tau_{b} \wedge t_{0}\right\}}\right] \\
= & I_{1}(x)+I_{2}(x)+I(x) .
\end{aligned}
$$

The terms $I_{1}(x)$ and $I_{2}(x)$ are exactly as in [19, Thm. 2]. However, the last expectation consists of two integrals, i.e. $I(x)=I_{3}(x)+I_{4}(x)$, because Parisian ruin allows the regulated risk process to go below zero, but not to stay there longer than $d$ since otherwise we will collect no dividends. Hence we additionally consider the term $I_{4}$, i.e. the case when the first claim is greater than $x+c s+\sigma y$. Furthermore, the function $I_{3}(x)$ is as in [19, Thm. 2] except that in addition $\lambda$ should be multiplied by $r$, because when the first claim arrives, the penalty with respect to the number of arrived claims gives an additional $r=r^{N_{T_{1}}}$.

Now, to prove the twice continuous differentiability of $v^{a, d}$ for all $x<a$, it is sufficient to show that $I_{4}(x)$ is twice continuously differentiable. Changing variables in (3.2) gives

$$
I_{4}(x)=\int_{0}^{t_{0}} \lambda r e^{-(\lambda+q) s} d s \int_{-b}^{b} H(b, s, y) d y \int_{0}^{\infty} \phi(-z, d) v^{a, d}(0) f(z+x+c s+\sigma y) d z
$$

Then from our assumption that the claim density $f$ is continuously differentiable and from the fact that $\phi$ is continuously differentiable (which follows from Lemma 3.7) it follows that $I_{4}(x)$ is twice continuously differentiable. Indeed, from the above equation one can compute that

$$
I_{4}^{\prime}(x)=\int_{0}^{t_{0}} \lambda r e^{-(\lambda+q) s} d s \int_{-b}^{b} H(b, s, y) d y \int_{0}^{\infty} \phi(-z, d) v^{a, d}(0) f^{\prime}(z+x+c s+\sigma y) d z
$$


Again, by changing variables we obtain

$$
\begin{aligned}
I_{4}^{\prime \prime}(x)= & \int_{0}^{t_{0}} \lambda r e^{-(\lambda+q) s} d s \\
& \times \int_{-b}^{b} H(b, s, y) d y \int_{z+x+c s+\sigma y}^{\infty}\left[\phi^{\prime}(z+x+c s+\sigma y, d) v^{a, d}(0) f^{\prime}(z)\right. \\
& \left.-\phi(0) v^{a, d}(0) f^{\prime}(z+x+c s+\sigma y)\right] d z .
\end{aligned}
$$

Finally, with a similar idea, equation (3.1) can be deduced from [19, Thm. 1] by changing the upper limit $u$ of integration to $\infty$ and multiplying $\lambda$ by an additional $r$ in the last part of equation (2) in [19, Thm. 1]. This completes the proof.

REMARK 3.2. Later, in Lemma 4.3 we prove that $v^{a, d} \in C^{2}(0, \infty)$ when $\sigma>0$ and $v^{a, d} \in C^{1}(0, \infty)$ if $\sigma=0$ and the density is absolutely continuous with respect to Lebesgue measure. This means that equation (3.1) holds true even under the weaker assumption that the jump density is absolutely continuous.

Now we will express the function $v^{a, d}$ in terms of some special function $h^{d}$ related to first passage times of the process $X$. Later, we will use two different methods to obtain an explicit expression for $h^{d}$. The first one uses the DicksonHipp operator (see Dickson and Hipp [10] and Li and Garrido [21]) and the second one uses fluctuation theory for Lévy processes.

Formally, let $y \in \mathbb{R}$ and define the first passage times of $X$ as follows:

$$
\tau_{y}^{+}:=\inf \left\{t \geqslant 0: X_{t} \geqslant y\right\}, \quad \tau_{y}^{-}:=\inf \left\{t \geqslant 0: X_{t} \leqslant y\right\} .
$$

For $x \leqslant a$ and $d \geqslant 0$, let

$$
h^{d}(x):=\mathbb{E}_{x}\left[r^{N_{\tau_{a}^{+}}} e^{-q \tau_{a}^{+}}, \tau_{a}^{+}<\tau^{d}\right] .
$$

We will write $h=h^{0}$ when $d=0$. Note that, for $\sigma=0$ and $d>0$, we have

$$
h^{d}(x)=0, \quad x<-c d,
$$

while

$$
\lim _{x \rightarrow-\infty} h^{d}(x)=0
$$

when $\sigma, d>0$.

Moreover, if $d=0$ and $\sigma>0$, we can observe that

$$
h^{d}(x)=h(x)=0, \quad x \leqslant 0,
$$

and if $d=\sigma=0$, then

$$
h^{d}(x)=h(x)=0, \quad x<0 .
$$


Using the fact that the process $X$ up-crosses all levels continuously, and the strong Markov property, we can derive the following representation of the function $v^{a, d}$.

LEMMA 3.3. The function $v^{a, d}$ satisfies

$$
v^{a, d}(x)= \begin{cases}h^{d}(x) v^{a, d}(a) & \text { if } x \leqslant a \\ x-a+v^{a, d}(a) & \text { if } x>a\end{cases}
$$

REMARK 3.4. By Lemmas 3.1 and 3.3 the function $h^{d}$, similarly to $v^{a, d}$, is twice differentiable and solves the same differential equation: for $x<a$ we have

$$
\frac{\sigma^{2}}{2}\left(h^{d}\right)^{\prime \prime}(x)+c\left(h^{d}\right)^{\prime}(x)-(\lambda+q) h^{d}(x)+\lambda r \int_{0}^{\infty} h^{d}(x-y) f(y) d y=0
$$

with the obvious boundary condition

$$
h^{d}(a)=1
$$

together with 3.4 - 3.7.

We will prove later that

$$
\left(v^{a, d}\right)^{\prime}(a)=1
$$

From (3.8) we can now deduce the following representation of the value function.

THEOREM 3.5. The function $v^{a, d}$ is given by

$$
v^{a, d}(x)= \begin{cases}\frac{h^{d}(x)}{\left(h^{d}\right)^{\prime}(a)} & \text { if } x \leqslant a, \\ x-a+\frac{1}{\left(h^{d}\right)^{\prime}(a)} & \text { if } x>a .\end{cases}
$$

The formal proof of (3.11) and hence of Theorem 3.5 is given in the Appendix.

Obviously, to maximize (3.12) we should choose $a$ that minimizes $\left(h^{d}\right)^{\prime}(a)$. This leads to the following statement.

THEOREM 3.6. If $h^{d} \in C^{2}(0, \infty)$ then the optimal barrier $a^{*}$ such that $v^{a^{*}, d}(x)=\max _{a \geqslant 0} v^{a, d}(x)$ solves

$$
\left(h^{d}\right)^{\prime \prime}\left(a^{*}\right)=0
$$

3.2. Identification of $h^{d}$. Now we will identify the function $h^{d}$ using two different techniques. The first one is to solve the equation (3.9) using the Dickson-Hipp operator. The second one uses fluctuation theory for spectrally negative Lévy processes. This allows finding the optimal function $v^{a, d}$ and the optimal strategy. 
3.2.1. The Dickson-Hipp operator method. The function $h^{d}$ will be given using the Dickson operators $T_{r}$ for $\mathfrak{R e}(r) \geqslant 0$ (see Dickson and Hipp [10] and Li and Garrido [21]). For any integrable real-valued function $g$ it is defined as

$$
T_{r} g(x):=\int_{x}^{\infty} e^{-r(u-x)} g(u) d u, \quad x \geqslant 0 .
$$

The operator $T_{r}$ has the following properties:

1. $T_{s} g(0)=\int_{0}^{\infty} e^{-s x} g(x) d x=\hat{g}(s)$, the Laplace transform of $g$;

2. $T_{r} T_{s} g(x)=\int_{x}^{\infty} e^{-r(y-x)} \int_{y}^{\infty} e^{-s(z-y)} g(z) d z d y$.

3. The operators $T_{s}$ pairwise commute, i.e. $T_{s} T_{r}=T_{r} T_{s}$, namely

$$
T_{s} T_{r} f(x)=T_{r} T_{s} f(x)=\frac{T_{s} f(x)-T_{r} f(x)}{r-s}, \quad s \neq r, x \geqslant 0 .
$$

More properties of the operators $T_{r}$ can be found in Li and Garrido [21].

Now let

$$
v_{y}(k, t)=\frac{d}{d t} V_{y}(k, t)
$$

with

$$
V_{y}(k, t)=\mathbb{P}\left(N_{\tau_{y}^{+}}=k, \tau_{y}^{+} \leqslant t \mid X_{0}=0\right), \quad k \in \mathbb{N}, y>0, t \geqslant 0 .
$$

LEMMA 3.7. If $\sigma>0$ then

$$
v_{y}(k, t)=\frac{\lambda^{k}}{k !} y t^{k-1} e^{-\lambda t} \int_{-\infty}^{\infty} \frac{1}{\sqrt{2 \pi t}} e^{-\frac{x^{2}}{2 t}} f^{k *}(c t+\sigma x-y) d x,
$$

and for $\sigma=0$,

$$
v_{y}(k, t)=\frac{\lambda^{k}}{k !} y t^{k-1} e^{-\lambda t} f^{k *}(c t-y),
$$

where $f^{k *}$ denotes the $k$ th convolution power of the claim density function.

The proof of this lemma is given in the Appendix.

Finaly, denote

$$
w_{d}(x):=\int_{0}^{\infty} \int_{0}^{d} e^{-q t} \sum_{k=0}^{\infty} r^{k} v_{y}(k, t) f(y+x) d t d y .
$$

The next theorem gives an explicit expression for the function $h^{d}$ producing the value function $v^{a, d}$ given in 3.12. 
THEOREM 3.8. The function $h^{d}$ can be expressed as follows:

1. for $\sigma=d=0$ and $0 \leqslant x \leqslant a$,

$$
h^{d}(x)=h(x)=\frac{\sum_{n=0}^{\infty}(\lambda r / c)^{n}\left(T_{\rho} f\right)^{* n} * \zeta(x)}{\sum_{n=0}^{\infty}(\lambda r / c)^{n}\left(T_{\rho} f\right)^{* n} * \zeta(a)} ;
$$

2. for $\sigma>0, d=0$ and $0<x \leqslant a$,

$$
h^{d}(x)=h(x)=\frac{\sum_{n=0}^{\infty}\left(2 \lambda r / \sigma^{2}\right)^{n}\left(\beta * T_{\rho} f\right)^{* n} * \zeta * \beta(x)}{\sum_{n=0}^{\infty}\left(2 \lambda r / \sigma^{2}\right)^{n}\left(\beta * T_{\rho} f\right)^{* n} * \zeta * \beta(a)} ;
$$

3. for $\sigma=0, d>0$ and $-c d \leqslant x \leqslant a$,

$$
h^{d}(x)=\frac{\sum_{n=0}^{\infty}(2 \lambda r / c)^{n}\left(T_{\rho} f\right)^{* n} * \varphi(x)}{\sum_{n=0}^{\infty}(2 \lambda r / c)^{n}\left(T_{\rho} f\right)^{* n} * \varphi(a)}
$$

4. for $\sigma, d>0$ and $x \leqslant a$,

$$
h^{d}(x)=\frac{\sum_{n=0}^{\infty}\left(2 \lambda r / \sigma^{2}\right)^{n}\left(\beta * T_{\rho} f\right)^{* n} * \varphi_{1}(x)}{\sum_{n=0}^{\infty}\left(2 \lambda r / \sigma^{2}\right)^{n}\left(\beta * T_{\rho} f\right)^{* n} * \varphi_{1}(a)},
$$

where

$$
\beta(x):=e^{-\left(\rho+2 c / \sigma^{2}\right) x}, \quad \zeta(x):=e^{\rho x}, \quad \varphi(x):=\zeta(x)-\frac{\lambda r}{c} \zeta * w_{d}(x),
$$

$\rho$ is the unique non-negative root of the Lundberg fundamental equation (3.24), and

$$
\varphi_{1}(x):=\left(\rho+\frac{2 c}{\sigma^{2}}\right) \zeta * \beta(x)+\beta(x)-\frac{2 \lambda r}{\sigma^{2}} \zeta * \beta * w_{d}(x) .
$$

Proof. For $\sigma \geqslant 0$ and $d \geqslant 0$ we take any $y<0$. Then from the strong Markov property we have

$$
\begin{aligned}
h^{d}(y) & =\mathbb{E}_{y}\left[r^{N_{\tau_{0}^{+}}} e^{-q \tau_{0}^{+}}, \tau_{0}^{+}<d\right] h^{d}(0)=\mathbb{E}_{0}\left[r^{N_{\tau_{-y}^{+}}} e^{-q \tau_{-y}^{+}}, \tau_{-y}^{+}<d\right] h^{d}(0) \\
& =h^{d}(0) \int_{0}^{d} e^{-q t} \sum_{k=0}^{\infty} r^{k} v_{-y}(k, t) d t .
\end{aligned}
$$

Then the equation 3.9 is equivalent to

$$
\begin{aligned}
& \frac{\sigma^{2}}{2}\left(h^{d}\right)^{\prime \prime}(x)+c\left(h^{d}\right)^{\prime}(x)-(\lambda+q) h^{d}(x)+\lambda r \int_{0}^{x} h^{d}(x-y) f(y) d y \\
& +\lambda r h^{d}(0) \int_{x}^{\infty} f(y) \int_{0}^{d} e^{-q t} \sum_{k=0}^{\infty} r^{k} v_{y-x}(k, t) d t d y=0 .
\end{aligned}
$$


To solve (3.21) we will follow the ideas given in [10] and [20]. We will focus mainly on cases 2 and 3 .

We start from case 2, that is, let $\sigma>0$ and $d=0$. Then (3.21) reduces to

$$
\frac{\sigma^{2}}{2}(h)^{\prime \prime}(x)+c(h)^{\prime}(x)-(\lambda+q) h(x)+\lambda r \int_{0}^{x} h(x-y) f(y) d y=0
$$

with the boundary conditions 3.6 and 3.10 . Taking the Laplace transforms of both sides of 3.22 we get

$$
\left(\frac{\sigma^{2}}{2} s^{2}+c s-(\lambda+q)-\lambda r \hat{f}(s)\right) \hat{h}(s)=\frac{\sigma^{2}}{2} s h(0)+\frac{\sigma^{2}}{2} h^{\prime}(0)+\operatorname{ch}(0),
$$

where $\hat{g}(s)$ is the Laplace transform of the function $g$. Let $\rho$ be the unique solution to the fundamental Lundberg equation (see Gerber and Shiu [12]):

$$
\frac{\sigma^{2}}{2} s^{2}+c s-(\lambda+q)+\lambda r \hat{f}(s)=0 .
$$

Then subtracting $\left[\frac{\sigma^{2}}{2} \rho^{2}+c \rho-(\lambda+q)+\lambda r \hat{f}(\rho)\right] \hat{h}(s)$ from the left side of 3.23 and dividing the resulting equation by $\left(\frac{\sigma^{2}}{2}(s+\rho)+c\right)(s-\rho)$ produces

$$
\begin{aligned}
\hat{h}(s)= & \frac{2 \lambda r / \sigma^{2}}{s+\rho+2 c / \sigma^{2}} \hat{\xi}(s) T_{s} T_{\rho} f(0)+\frac{\left(\rho+2 c / \sigma^{2}\right) h(0)+h^{\prime}(0)}{(s-\rho)\left(s+\rho+2 c / \sigma^{2}\right)} \\
& +\frac{h(0)}{s+\rho+2 c / \sigma^{2}} .
\end{aligned}
$$

Inverting the above Laplace transforms gives

$$
\begin{aligned}
h(x)= & \frac{2 \lambda r}{\sigma^{2}} \int_{0}^{x} h(x-y) \beta * T_{\rho} f(y) d y \\
& +\left[\left(\rho+2 c / \sigma^{2}\right) h(0)+h^{\prime}(0)\right] \zeta * \beta(x)+h(0) \beta(x) .
\end{aligned}
$$

On the other hand, we know that the general solution of $(3.22)$ is

$$
h(x)=\eta_{1} h_{1}(x)+\eta_{2} h_{2}(x) \quad \text { for } 0 \leqslant x \leqslant a,
$$

where $h_{1}(x)$ and $h_{2}(x)$ are two linearly independent particular solutions of 3.22 and $\eta_{1}, \eta_{2}$ are any real numbers. One can find two linearly independent solutions $h_{1}(x)$ and $h_{2}(x)$ by specifying the initial conditions:

$$
\left\{\begin{array}{lll}
h_{1}(0)=1 & \text { and } \quad & h_{1}^{\prime}(0)=-\left(\rho+2 c / \sigma^{2}\right) \\
h_{2}(0)=0 & \text { and } \quad & h_{2}^{\prime}(0)=1
\end{array}\right.
$$


These conditions together with (3.6) and 3.10 give $h(x)=h_{2}(x) / h_{2}(a)$. Since $h_{2}(x)$ is a particular solution of 3.22 , from 3.25) with the boundary conditions $h_{2}(0)=0$ and $h_{2}^{\prime}(0)=1$ we have

$$
\begin{aligned}
h_{2}(x) & =\frac{2 \lambda r}{\sigma^{2}} \int_{0}^{x} h(x-y) \beta * T_{\rho} f(y) d y+\zeta * \beta(x) \\
& =\sum_{n=0}^{\infty}\left(\frac{2 \lambda r}{\sigma^{2}}\right)^{n}\left(\beta * T_{\rho} f\right)^{* n} * \zeta * \beta(x) .
\end{aligned}
$$

Finally, we conclude that

$$
h(x)=\frac{\sum_{n=0}^{\infty}\left(2 \lambda r / \sigma^{2}\right)^{n}\left(\beta * T_{\rho} f\right)^{* n} * \zeta * \beta(x)}{\sum_{n=0}^{\infty}\left(2 \lambda r / \sigma^{2}\right)^{n}\left(\beta * T_{\rho} f\right)^{* n} * \zeta * \beta(a)} .
$$

To analyze case 3 we assume that $\sigma=0$ and $d>0$. Since $\mathbb{P}\left(\tau_{a}^{+}<\tau^{d}\right)>0$, the definition of $h^{d}(x)$ yields $h^{d}(0) \neq 0$. Dividing both sides of 3.21$)$ by $h^{d}(0)$ and letting $\xi(x)=h^{d}(x) / h^{d}(0)$ produces

$$
c \xi^{\prime}(x)-(\lambda+q) \xi(x)+\lambda r \int_{0}^{x} \xi(x-y) f(y) d y+\lambda r w_{d}(x)=0,
$$

where $w_{d}(x)$ is given in 3.16. Taking the Laplace transforms of both sides of (3.26, for sufficiently large $s$ we get

$$
c s \hat{\xi}(s)-c \xi(0)-(\lambda+q) \hat{\xi}(s)+\lambda r \hat{f}(s) \hat{\xi}(s)+\lambda r \hat{w}_{d}(s)=0,
$$

which after rearranging terms and using the fact that $\xi(0)=1$ leads to

$$
[c s-(\lambda+q)+\lambda r \hat{f}(s)] \hat{\xi}(s)=c-\lambda r \hat{w}_{d}(s) .
$$

Recall that $\rho$ is the unique non-negative root of the Lundberg fundamental equation 3.24 with $\sigma=0$. Then subtracting $[c \rho-(\lambda+q)+\lambda r \hat{f}(\rho)] \hat{\xi}(s)$ from the left side of 3.27) and dividing the resulting equation by $s-\rho$ produces

$$
\hat{\xi}(s)=\frac{\lambda r}{c} \hat{\xi}(s) T_{s} T_{\rho} f(0)+\frac{1}{s-\rho}-\frac{\lambda r \hat{w}_{d}(s)}{(s-\rho) c} .
$$

Inverting the Laplace transforms in 3.28 gives

$$
\xi(x)=\frac{\lambda r}{c} \int_{0}^{x} \xi(x-y) T_{\rho} f(y) d y+e^{\rho x}-\frac{\lambda r}{c} \zeta * w_{d}(x)=\sum_{n=0}^{\infty}\left(\frac{\lambda r}{c}\right)^{n} * \varphi(x) .
$$

Since

$$
h^{d}(x)=h^{d}(0) \xi(x)=h^{d}(0)\left[\sum_{n=0}^{\infty}\left(\frac{\lambda r}{c}\right)^{n} * \varphi(x)\right],
$$


from the boundary condition 3.10 we get

$$
h^{d}(0)=\frac{1}{\sum_{n=0}^{\infty}(\lambda r / c)^{n} * \varphi(a)} .
$$

Then (3.29) produces 3.19), which completes the proof of case 3 .

Cases 1 and 4 could be analyzed analogously. More precisely, in case 1 equation (3.21) reduces to

$$
c \xi^{\prime}(x)-(\lambda+q) \xi(x)+\lambda r \int_{0}^{x} \xi(x-y) f(y) d y=0 .
$$

In the next step we take the Laplace transforms of both sides of this equation. Then we again take $\rho$ as the unique non-negative root of the Lundberg fundamental equation. In the next steps we subtract $[c \rho-(\lambda+q)+\lambda r \hat{f}(\rho)] \hat{\xi}(s)$, divide the result by $s-\rho$ and finally invert the Laplace transform. Using the boundary condition 3.10 produces 3.17.

Similarly, to get the result of case 4 we divide both sides of 3.21 by $h^{d}(0)>0$ and consider $\xi(x)=h^{d}(x) / h^{d}(0)$. Then we again take the Laplace transforms of both sides and subtract $\left[\frac{\sigma^{2}}{2} \rho^{2}+c \rho-(\lambda+q)+\lambda r \hat{f}(\rho)\right] \hat{\xi}(s)$, where again $\rho$ is the unique non-negative root of the Lundberg fundamental equation. Finally, we divide the result by $\left(\frac{\sigma^{2}}{2}(s+\rho)+c\right)(s-\rho)$ and by inverting the Laplace transforms and by using the boundary conditions (3.10) and (3.5) we end up with 3.20 .

3.2.2. Fluctuation theory method. Before starting the identification of the functions $h^{d}$, we introduce a little extra notation and background on spectrally negative Lévy processes. We say that $X=\left\{X_{t}, t \geqslant 0\right\}$ is a spectrally negative Lévy process on the filtered probability space $\left(\Omega, \mathcal{F},\left\{\mathcal{F}_{t}: t \geqslant 0\right\}, \mathbb{P}\right)$ if $X$ is a stochastic process issued from the origin which has stationary and independent increments and càdlàg paths that have no positive jump discontinuities. One can easily verify that process $(2.1)$ is an example.

The Laplace exponent of $X$ is denoted by $\psi(\theta)$, i.e.

$$
\psi(\theta) t=\log \mathbb{E}\left[e^{\theta X_{t}}\right],
$$

which is well defined for $\theta \geqslant 0$. This allows us to define, for $q \geqslant 0$, the largest $\operatorname{root} \Phi(q)$ of the equation $\psi(\theta)=q$ :

$$
\Phi(q)=\sup \{\theta \geqslant 0: \psi(\theta)=q\} .
$$

We further introduce the $q$-scale function $W^{(q)}$ of $X$, which is the strictly increasing, continuous function uniquely defined on $[0, \infty)$ through its Laplace transform which is given by

$$
\int_{0}^{\infty} e^{-\theta x} W^{(q)}(x) d x=\frac{1}{\psi(\theta)-q}, \quad \theta>\Phi(q) .
$$


We extend $W^{(q)}$ to the whole real line by setting $W^{(q)}(x)=0$ for $x<0$. We write $W=W^{(0)}$ when $q=0$. We will also use the following results for the classical ruin and Parisian ruin probability (for details see [15] and [24]):

$$
\begin{aligned}
& \mathbb{P}_{x}\left(\tau^{0}=\infty\right)=\mathbb{E}\left[X_{1}\right] W(x) \\
& \mathbb{P}_{x}\left(\tau^{d}=\infty\right)=\mathbb{E}\left[X_{1}\right] \frac{\int_{0}^{\infty} W(x+z) z \mathbb{P}\left(X_{d} \in d z\right)}{\int_{0}^{\infty} z \mathbb{P}\left(X_{d} \in d z\right)}
\end{aligned}
$$

The basic observation used in this section is that by 2.1 the process

$$
Z_{t}^{\alpha}:=\alpha X_{t}+\log (r) N_{t}=\alpha x+\alpha c t-\sum_{i=1}^{N_{t}}\left(\alpha C_{i}-\log (r)\right)+\alpha \sigma B_{t}
$$

is again a Lévy processes. Hence from the Lévy-Khinchin theorem there exists a function $\psi_{r}(\alpha)$ such that

$$
\log \mathbb{E}\left[e^{\alpha X_{t}+\log (r) N_{t}}\right]=\log \mathbb{E}\left[r^{N_{t}} e^{\alpha X_{t}}\right]=\psi_{r}(\alpha) t .
$$

Moreover,

$$
\begin{aligned}
\psi_{r}(\alpha) & =c \alpha+\frac{1}{2} \sigma^{2} \alpha^{2}+\lambda r \int_{0}^{\infty} e^{-\alpha z} f(z) d z-\lambda \\
& =c \alpha+\frac{1}{2} \sigma^{2} \alpha^{2}+\lambda r \int_{0}^{\infty} e^{-\alpha z} f(z) d z-\lambda r-\lambda(1-r)
\end{aligned}
$$

and hence $\psi_{r}$ is the Laplace exponent of the Lévy process

$$
X_{t}^{r}:=x+c t-\sum_{i=1}^{N_{t}^{r}} C_{i}+\sigma B_{t}
$$

killed with fixed intensity $\lambda(1-r)$, where $N_{t}^{r}$ is a Poisson process with intensity $\lambda r$. Note also that for fixed $r$ the function $\alpha \mapsto \psi_{r}(\alpha)$ is continuous and goes to infinity as $\alpha$ tends to infinity.

Thus there exists $\Phi_{r}(q)$ such that

$$
\psi_{r}\left(\Phi_{r}(q)\right)=q
$$

Moreover, we can define a new scale function $W_{r}^{(q)}:[0, \infty) \rightarrow[0, \infty)$ for $\psi_{r}$ as follows:

$$
\int_{0}^{\infty} e^{-\theta y} W_{r}^{(q)}(y) d y=\frac{1}{\psi_{r}(\theta)-q}, \quad \theta>\Phi_{r}(q)
$$


We will also use the following classical change of measure for the process $Z_{t}^{\alpha}$ given in 3.32):

$$
\left.\frac{d \mathbb{P}^{\alpha, r}}{d \mathbb{P}}\right|_{\mathcal{F}_{t}}=r^{N_{t}} \exp \left(\alpha X_{t}-\psi_{r}(\alpha) t\right)=\exp \left(Z_{t}^{\alpha}-\psi_{r}(\alpha) t\right) .
$$

Under the measure $\mathbb{P}^{\alpha, r}$ the process $Z^{\alpha}$ is still a Lévy process with the new triple (see Palmowski and Rolski [26] and Kyprianou [16] for details). We will use the above change of measure with $\alpha=\Phi_{r}(q)$ defined in (3.35). Observe that for this choice of $\alpha$ the function $W_{r}^{(q)}(x)$ defined formally in 3.36 equals $e^{\Phi_{r}(q) x} W_{r}^{(0)}(x)$ for $W_{r}^{(0)}(x)$ calculated under the new measure $\mathbb{P}^{\Phi_{r}(q), r}$.

We will express $h^{d}(x)$ in terms of the above modified scale function $W_{r}^{(q)}(x)$. This can be seen as solving the Parisian-type two-sided exit problem penalized by the number of claims.

THEOREM 3.9. For any $\sigma \geqslant 0$ the function $h^{d}(x)$ can be expressed as follows:

$$
h^{d}(x)=\mathbb{E}_{x}\left[r^{N_{\tau_{a}^{+}}} e^{-q \tau_{a}^{+}}, \tau_{a}^{+}<\tau^{d}\right]=\frac{\int_{0}^{\infty} W_{r}^{(q)}(x+z) z \mathbb{P}\left(X_{d} \in d z\right)}{\int_{0}^{\infty} W_{r}^{(q)}(a+z) z \mathbb{P}\left(X_{d} \in d z\right)}
$$

and

$$
h(x)=h^{0}(x)=\mathbb{E}_{x}\left[r^{N_{\tau_{a}^{+}}} e^{-q \tau_{a}^{+}}, \tau_{a}^{+}<\tau^{0}\right]=\frac{W_{r}^{(q)}(x)}{W_{r}^{(q)}(a)} .
$$

Proof. We will use similar arguments to those used for the classical two-sided upward exit problem for a spectrally negative Lévy process (see Kyprianou and Palmowski [17]). First we apply the change of measure (3.37) together with the Optional Stopping Theorem and use the crucial observation that $X\left(\tau_{a}^{+}\right)=a$ (since $X$ has no strictly positive jumps) to conclude that

$$
h^{d}(x)=\mathbb{E}_{x}\left[r^{N_{\tau_{a}^{+}}} e^{-q \tau_{a}^{+}}, \tau_{a}^{+}<\tau^{d}\right]=e^{\Phi_{r}(q)(x-a)} \mathbb{P}_{x}^{\Phi_{r}(q), r}\left(\tau_{a}^{+}<\tau^{d}\right) .
$$

Then from the strong Markov property we can derive the following representation:

$$
h^{d}(x)=\mathbb{E}_{x}\left[r^{N_{\tau_{a}^{+}}} e^{-q \tau_{a}^{+}}, \tau_{a}^{+}<\tau^{d}\right]=e^{\Phi_{r}(q)(x-a)} \frac{\mathbb{P}_{x}^{\Phi_{r}(q), r}\left(\tau^{d}=\infty\right)}{\mathbb{P}_{a}^{\Phi_{r}(q), r}\left(\tau^{d}=\infty\right)} .
$$

The identity (3.38) together with 3.31 completes the proof.

\section{OPTIMALITY}

We will now give the main results of this paper which give sufficient conditions for optimality of the barrier strategy $\pi_{a^{*}}$.

DEFINITION 4.1. We say that a function $g$ is sufficiently smooth whenever $g \in C^{1}(0, \infty)$ if $\sigma=0$ and $g \in C^{2}(0, \infty)$ if $\sigma>0$. 
Let $\Gamma$ be an extended generator of $X_{t}$ defined by

$$
\Gamma g(x)=c g^{\prime}(x)+\frac{\sigma^{2}}{2} g^{\prime \prime}(x)-\lambda g(x)+\lambda r \int_{(0, \infty)} g(x-y) f(y) d y
$$

acting on sufficiently smooth functions.

We will start from the classical Verification Lemma.

THEOREM 4.2. Suppose $\pi$ is an admissible dividend strategy such that $v^{\pi}$ is sufficiently smooth and for all $x>0$,

$$
\max \left\{(\Gamma-q) v^{\pi}(x), 1-\left(v^{\pi}\right)^{\prime}(x)\right\} \leqslant 0 .
$$

Then $v^{\pi}(x)=v^{*}(x)$ for all $x \in \mathbb{R}$.

REMARK. The inequality (4.1) is called the Hamilton-Jacobi-Bellman system and is classical in all stochastic optimization problems.

Proof of Theorem 4.2 We follow classical arguments. Suppose that $g$ is is sufficiently smooth and that

$$
\max \left\{(\Gamma-q) g(x), 1-g^{\prime}(x)\right\} \leqslant 0 .
$$

We will prove that

$$
g(x) \geqslant \sup _{\pi \in \Pi} v^{\pi}(x), \quad x \in \mathbb{R} .
$$

Having a strategy $\pi^{*}$ for which $g(x)=v^{\pi^{*}, d}(x)$ will complete the proof. To prove 4.2. we will consider the Markov process $\left(t, N_{t}, X_{t}, \varsigma_{t}^{X}\right)$ with

$$
\varsigma_{t}^{Z}=t-\sup \left\{s \leqslant t: Z_{t} \geqslant 0\right\}
$$

for some process $Z$. By Sato [28, Ch. 6, Thm. 31.5] the function $\varpi(t, k, x, z):=$ $r^{k} e^{-q t} g(x) \mathbf{1}_{\{z \leqslant r\}}$ is in the domain of the extended generator $\mathcal{A}$ of this four-dimensional process. In fact using similar arguments to those in deriving (3.1) from the definition of the infinitesimal generator, one can prove that

$$
(\mathcal{A}-q \mathbb{I}) \varpi(t, k, x, z)=r^{k}(\Gamma-q) g(x) .
$$

Recall that $U_{t}^{\pi}=X_{t}-L_{t}^{\pi}$ and note that the regulations $L_{t}^{\pi}$ do not modify the jump process of $X$. Note that a finite number of discontinuities of $g$ and hence also a single discontinuity of $\mathbf{1}_{\{z \leqslant r\}}$ are allowed here. Hence we can apply Itô's lemma and letting $U=U^{\pi}$ and $L=L^{\pi}$ we derive

$$
\begin{aligned}
e^{-q t} r^{N_{t}} g\left(U_{t}\right) \mathbf{1}_{\left\{\varsigma_{t}^{U} \leqslant r\right\}}-g\left(U_{0}\right)= & r^{N_{t}} J_{g}(t)-r^{N_{t}} \int_{0}^{t} e^{-q s} g^{\prime}\left(U_{s^{-}}\right) d L_{s}^{c} \\
& +\int_{0}^{t} e^{-q s} r^{N_{s}}(\Gamma g-q g)\left(U_{s-}\right) d s+M_{t},
\end{aligned}
$$


where $M_{t}$ is a local martingale with $M_{0}=0, L^{c}$ is the pathwise continuous part of $L$ and

$$
J_{g}(t)=\sum_{s \leqslant t} e^{-q s}\left[g\left(A_{s}+B_{s}\right)-g\left(A_{s}\right)\right] \mathbf{1}_{\left\{B_{s} \neq 0\right\}},
$$

where $A_{s}=U_{s-}+\Delta X_{s}$ with $\Delta X_{s}=X_{s}-X_{s-}$, and $B_{s}=-\Delta L_{s}$ denotes the jump of $-L$ at time $s$. Let $T_{n}$ be a localizing sequence of $M$. Applying the Optional Stopping Theorem to the stopping times $T_{k}^{\prime}=T_{k} \wedge \tau^{\pi, d}$ and using the Fatou Theorem we derive

$$
\begin{aligned}
& g(x) \geqslant \mathbb{E}_{x} r^{N_{T_{n}^{\prime}}}\left[e^{-q T_{n}^{\prime}} g\left(U_{T_{n}^{\prime}}\right) \mathbf{1}_{\left\{\varsigma_{T_{n}^{\prime}}^{U} \leqslant r\right\}}-J_{g}\left(T_{n}^{\prime}\right)\right]+\mathbb{E}_{x} r^{N_{T_{n}^{\prime}}} \int_{0}^{T_{n}^{\prime}} e^{-q s} g^{\prime}\left(U_{s^{-}}\right) d L_{s}^{c} \\
& -\mathbb{E}_{x} \int_{0}^{T_{n}^{\prime}} r^{N_{s}} e^{-q s}(\Gamma g-q g)\left(U_{s-}\right) d s
\end{aligned}
$$

Invoking the variational inequalities $g^{\prime}(x) \geqslant 1$ (hence $g\left(A_{s}+B_{s}\right)-g\left(A_{s}\right) \leqslant$ $-\Delta L_{s}$ if $\left.A_{s}>0\right)$ and $(\Gamma-q) g(x) \leqslant 0$ we have

$$
\begin{aligned}
g(x) \geqslant & \mathbb{E}_{x} e^{-q T_{n}^{\prime}} r^{N_{T_{n}^{\prime}}} g\left(U_{T_{n}^{\prime}}\right) \mathbf{1}_{\left\{\varsigma_{T_{n}^{\prime}}^{U} \leqslant d\right\}}+\mathbb{E}_{x} r^{N_{T_{n}^{\prime}}} \int_{0}^{T_{n}^{\prime}} e^{-q s} d L_{s} \\
\geqslant & \mathbb{E}_{x}\left[r^{N_{\tau^{\pi, d}}} e^{-q \tau^{\pi, d}} g\left(U_{\tau^{\pi, d}}\right), \tau^{\pi, d} \leqslant T_{n}^{\prime}\right] \\
& +\mathbb{E}_{x} r^{N_{\tau^{\pi, d}}}\left[\int_{0}^{\tau^{\pi, d}} e^{-q s} d L_{s}, \tau^{\pi, d} \leqslant T_{n}^{\prime}\right] .
\end{aligned}
$$

Letting $n \rightarrow \infty$ and using the monotone convergence theorem and the fact that $\mathbf{1}_{\left\{\varsigma_{\tau, d}^{U} \leqslant d\right\}}=0$ completes the proof.

We will now focus on the optimality of the barrier strategy $\pi^{a^{*}}$.

LEMMA 4.3. The function $v^{a, d}$ is sufficiently smooth.

Proof. From Theorem 3.5 it follows that it sufficient to prove that $h^{d}$ is sufficiently smooth or by Theorem 3.9 that $W_{r}^{(q)}$ is sufficiently smooth. The function $W_{r}^{(q)}$ is just the scale function for the Lévy process $X_{t}^{r}$. Hence from Kyprianou et al. [15, Lem. 2.4, p. 117], if $\sigma=0$ and the Lévy measure is absolutely continuous with respect to the Lebesgue measure, then $W_{r}^{(q)} \in C^{1}(0, \infty)$. Moreover, if $\sigma>0$ then by [15, Thm. 3.10, p. 136] we have $W_{r}^{(q)} \in C^{2}(0, \infty)$.

THEOREM 4.4. If

$$
(\Gamma-q) h^{d}(x) \leqslant 0 \quad \text { for } x \geqslant a^{*},
$$

then the barrier strategy $\pi^{a^{*}}$ is optimal. 
Proof. Note that from 3.9 it follows that

$$
(\Gamma-q) h^{d}(x)=0 \quad \text { for } x \leqslant a^{*} .
$$

From the choice of $a^{*}$ and Theorem 3.5 it follows that $\left(v^{a^{*}, d}\right)^{\prime}(x) \leqslant 1$. This completes the proof in view of the Verification Lemma 4.2.

Moreover, we can give another sufficient condition for the barrier strategy to be optimal.

COROllary 4.5. Suppose that

$$
\left(h^{d}\right)^{\prime}(a) \leqslant\left(h^{d}\right)^{\prime}(b) \quad \text { for all } a^{*} \leqslant a \leqslant b .
$$

Then the barrier strategy $\pi_{a^{*}}$ at $a^{*}$ is an optimal strategy.

Proof. With the use of Theorem 4.4 and (4.6), the proof is the same as that of [22, Theorem 2].

THEOREM 4.6. Suppose that the claims $C_{i}(i=1,2, \ldots)$ have density $f$ with $f^{\prime}$ decreasing. Then $v^{a^{*}, d}=v^{*}$ and $\pi_{a^{*}}$ is an optimal strategy.

Proof. The proof follows the same idea as the proof of [7, Corollary 5.2] and it is based on Theorem 3.9 .

\section{EXAMPLES}

In this section we consider the case when the claim sizes are exponentially distributed with parameter $\mu>0$, that is, $f(x)=\mu e^{-\mu x}$ for $x>0$. Moreover, we present a formula for the function $h^{d}$ in case $\sigma>0$ determined by the second method (i.e. via change of measure) and a formula in case $\sigma=0$ determined by the Dickson-Hipp operator method.

1. Assume that $\sigma>0$. One can compute that

$$
\psi_{r}(\theta)=\frac{\sigma^{2}}{2} \theta^{2}+c \theta+\lambda r \frac{\mu}{\theta+\mu}-\lambda
$$

Now using the same argument as in [15] we can prove that the equation $\psi_{r}(\theta)=q$ has exactly three real solutions $\Phi_{r}(q), \varpi_{1}(q), \varpi_{2}(q)$, which satisfy $-\varpi_{1}(q)<$ $-\mu<-\varpi_{2}(q)<0 \leqslant \Phi_{r}(q)$. Then

$$
W_{r}^{(q)}(x)=\frac{e^{\Phi_{r}(q) x}}{\psi_{r}^{\prime}\left(\Phi_{r}(q)\right)}+\frac{e^{-\varpi_{1}(q) x}}{\psi_{r}^{\prime}\left(-\varpi_{1}(q)\right)}+\frac{e^{-\varpi_{2}(q) x}}{\psi_{r}^{\prime}\left(-\varpi_{2}(q)\right)}
$$

and

$$
\mathbb{P}\left(X_{d} \in d z\right)=e^{-\mu d} \sum_{k=0}^{\infty} \frac{(\mu d)^{k}}{k !} \int_{0}^{\infty} \frac{\mu y^{k-1} e^{-\mu y}}{(k-1) !} \mathcal{N}((d z+y-c d) \sigma \sqrt{d}) d y
$$


where $\mathcal{N}$ is a standard normal distribution function. The above formulas together with Theorem 3.9 give the value function 3.12.

2. Assume that $\sigma=0$. Since $f$ is an exponential density, $T_{\rho} f(x)$ becomes

$$
T_{\rho} f(x)=\int_{x}^{\infty} e^{-\rho(y-x)} \mu e^{-\mu y} d y=\frac{\mu}{\rho+\mu} e^{-\mu x} .
$$

Then

$$
\left(T_{\rho} f\right)^{* n}(x)=\left(\frac{\mu}{\rho+\mu}\right)^{n} \frac{x^{n-1}}{(n-1) !} e^{-\mu x}, \quad n>1
$$

and

$$
w_{d}(x)=e^{-\mu x} u(d), \quad \text { where } \quad u(d)=\sum_{k=0}^{\infty} \frac{r^{k} \lambda^{k}(\mu c)^{k+1}}{k !(k+1) !} \int_{0}^{d} t^{2 k} e^{-(\lambda+q+\mu c) t} d t .
$$

Substituting (5.1) and (5.2) into (3.17) and (3.19), respectively, gives

$$
h^{d}(x)= \begin{cases}\frac{\vartheta(x)}{\vartheta(a)}, & d=0 \text { and } 0 \leqslant x \leqslant a, \\ \frac{\varrho(x)}{\varrho(a)}, & d>0 \text { and }-c d \leqslant x \leqslant a,\end{cases}
$$

where

$$
\begin{aligned}
\vartheta(x)= & \sum_{n=1}^{\infty} \frac{(\lambda r \mu)^{n}}{(n-1) ! c^{n}(\rho+\mu)^{n}} e^{\rho x} \int_{0}^{x} y^{n-1} e^{-(\rho+\mu) y} d y+e^{\rho x}, \\
\varrho(x)= & \sum_{n=1}^{\infty} \frac{(\lambda r \mu)^{n}}{(n-1) ! c^{n}(\rho+\mu)^{n}} \\
& \times\left[\left(1-\frac{\lambda r u(d)}{c(\rho+\mu)}\right) e^{\rho x} \int_{0}^{x} y^{n-1} e^{-(\rho+\mu) y} d y+\frac{\lambda r, u(d)}{c n(\rho+\mu)} x^{n} e^{-\mu x}\right] \\
& +e^{\rho x}-\frac{\lambda r u(d)}{c(\rho+\mu)}\left(e^{\rho x}-e^{-\mu x}\right) .
\end{aligned}
$$

Moreover,

$$
\vartheta^{\prime}(x)=\sum_{n=1}^{\infty} \frac{(\lambda r \mu)^{n}}{(n-1) ! c^{n}(\rho+\mu)^{n}}\left[\rho e^{\rho x} \int_{0}^{x} y^{n-1} e^{-(\rho+\mu) y} d y+x^{n-1} e^{-\mu x}\right]+\rho e^{\rho x}
$$

and

$$
\begin{aligned}
& \varrho^{\prime}(x)=\sum_{n=1}^{\infty} \frac{(\lambda r \mu)^{n}}{(n-1) ! c^{n}(\rho+\mu)^{n}} \\
& \times\left[\left(1-\frac{\lambda r u(d)}{c(\rho+\mu)}\right) \rho e^{\rho x} \int_{0}^{x} y^{n-1} e^{-(\rho+\mu) y} d y+x^{n-1} e^{-\mu x}-\frac{\lambda \mu r u(d)}{c n(\rho+\mu)} x^{n} e^{-\mu x}\right] \\
& +\rho e^{\rho x}-\frac{\lambda r u(d)}{c(\rho+\mu)}\left(\rho e^{\rho x}+\mu e^{-\mu x}\right) .
\end{aligned}
$$


Further, by differentiating we can get expressions for $\vartheta^{\prime \prime}(x)$ and $\varrho^{\prime \prime}(x)$. Then solving the equations $\vartheta^{\prime \prime}(x)=0$ and $\varrho^{\prime \prime}(x)=0$ we will derive the optimal barrier level $a^{*}$ for $d=0$ and $d>0$, respectively.

To sum up, for $d=0$ we have

$$
v^{a^{*}, d}(x)= \begin{cases}\frac{\vartheta(x)}{\vartheta^{\prime}\left(a^{*}\right)} & \text { if } 0 \leqslant x \leqslant a^{*}, \\ x-a^{*}+\frac{\vartheta\left(a^{*}\right)}{\vartheta^{\prime}\left(a^{*}\right)} & \text { if } x>a^{*},\end{cases}
$$

and for $d>0$,

$$
v^{a^{*}, d}(x)= \begin{cases}\frac{\varrho(x)}{\varrho^{\prime}\left(a^{*}\right)} & \text { if }-c d \leqslant x \leqslant a^{*}, \\ x-a^{*}+\frac{\varrho\left(a^{*}\right)}{\varrho^{\prime}\left(a^{*}\right)} & \text { if } x>a^{*} .\end{cases}
$$

5.1. Numerical analysis. Using the above expressions we were able to find the function $h^{d}(x)$ numerically and hence the value function. We also identified the optimal barriers. This shows that the algorithms presented can be used in practice.

Let $\lambda=10, \mu=1, c=15, q=0.1, r=0.8$. We will consider two cases: $d=0$ and $d=2$. The positive root of (3.24) is $\rho=0.24493$. For $d=0$ solving $\vartheta^{\prime \prime}(x)=0$ produces $a^{*} \approx 0.7693$ and for $d=2$ solving $\varrho^{\prime \prime}(x)=0$ gives $a^{*} \approx 0.52202$. In Figures 3 and 5 we show what $v^{a^{*}, d}$ looks like. With the help of

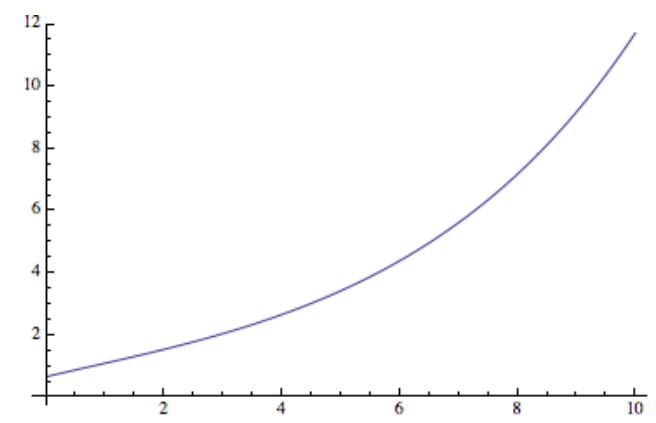

Figure 3. $v^{a^{*}, d}$ for $\lambda=10, \mu=1, c=15, q=0.1, r=0.8$ and $d=0$.

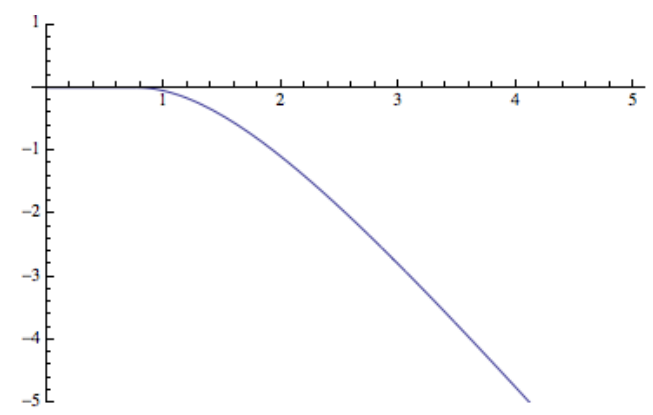

FiguRE 4. $(\Gamma-q) v^{a^{*}, d}(x)$ for $\lambda=10, \mu=1, c=15, q=0.1, r=0.8$ and $d=0$. 


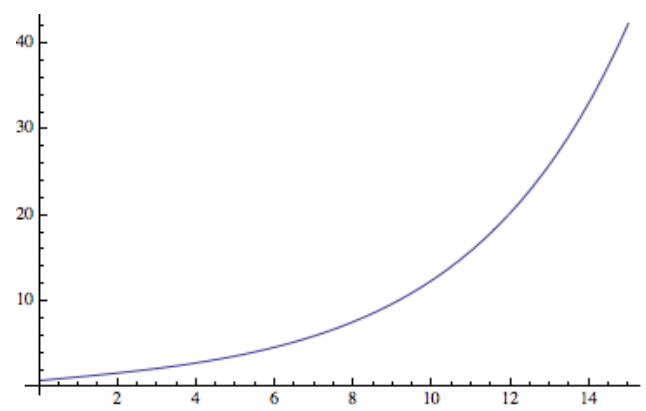

FiguRE 5. $v^{a^{*}, d}$ for $\lambda=10, \mu=1, c=15, q=0.1, r=0.8$ and $d=2$.

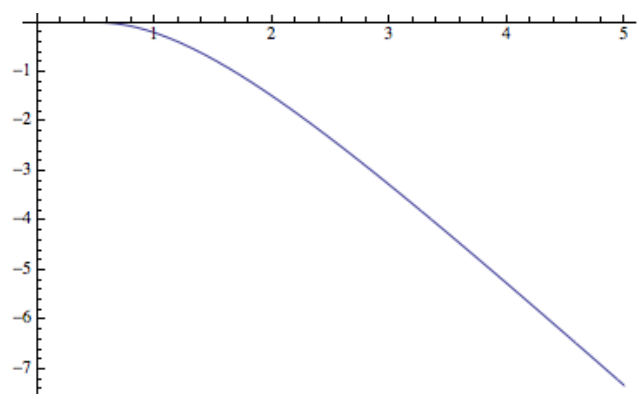

FiguRE 6. $(\Gamma-q) v^{a^{*}, d}(x)$ for $\lambda=10, \mu=1, c=15, q=0.1, r=0.8$ and $d=2$.

Mathematica we also plotted $(\Gamma-q) v^{a^{*}, d}(x)$ (see Figures 4 and 6). In all cases we have $(\Gamma-q) v^{a^{*}, d}(x) \leqslant 0$, hence by Theorem 4.4 the barrier $a^{*}$ produces the optimal strategy in both cases (this is also straightforward from Theorem 4.6 and the choice of the exponential claim size).

\section{APPENDIX}

Proof of Theorem 3.5 Observe that the process given in 2.1 is a spectrally negative Lévy process. Moreover, it is a Markov process. Since this process upcrosses all levels continuously, from the Markov property it follows that for general $b>a$,

$$
\begin{aligned}
\mathbb{E}_{x}\left[r^{N_{\tau_{b}^{+}}} e^{-q \tau_{b}^{+}}, \tau_{b}^{+}\right. & \left.<\tau^{d}\right] \\
& =\mathbb{E}_{x}\left[r^{N_{\tau_{a}^{+}}} e^{-q \tau_{a}^{+}}, \tau_{a}^{+}<\tau^{d}\right] \mathbb{E}_{a}\left[r^{N_{\tau_{b}^{+}}} e^{-q \tau_{b}^{+}}, \tau_{b}^{+}<\tau^{d}\right] .
\end{aligned}
$$

Taking $g^{d}(x):=\mathbb{E}_{x}\left[r^{N_{\tau_{b}^{+}}} e^{-q \tau_{b}^{+}}, \tau_{b}^{+}<\tau^{d}\right]$ we have

$$
h^{d}(x)=\frac{g^{d}(x)}{g^{d}(a)} .
$$


In fact for any $x<y$ we have

$$
\mathbb{E}_{x}\left[r^{N_{\tau_{y}^{+}}} e^{-q \tau_{y}^{+}}, \tau_{y}^{+}<\tau^{d}\right]=\frac{g^{d}(x)}{g^{d}(y)} .
$$

Note that for $n \in \mathbb{N}$,

$$
\begin{aligned}
v^{a, d}(a) & \geqslant \mathbb{E}_{a}\left[r^{N_{\tau_{a+1 / n}^{+}}} e^{-q \tau_{a+1 / n}^{+}}, \tau_{a+1 / n}^{+}<\tau^{d}\right] v^{a, d}\left(a+\frac{1}{n}\right) \\
& =\mathbb{E}_{a}\left[r^{{ }^{N} \tau_{a+1 / n}^{+}} e^{-q \tau_{a+1 / n}^{+}}, \tau_{a+1 / n}^{+}<\tau^{d}\right]\left(v^{a, d}(a)+\frac{1}{n}\right)
\end{aligned}
$$

and

$$
\begin{aligned}
v^{a, d}(a) \leqslant & \mathbb{E}_{a}\left[r^{N_{\tau_{a+1 / n}^{+}}} e^{-q \tau_{a+1 / n}^{+}}, \tau_{a+1 / n}^{+}<\tau^{d}\right]\left(v^{a, d}(a)+\frac{1}{n}\right) \\
& +\frac{1}{n} \mathbb{E}_{a}\left[r^{N_{\tau_{a+1 / n}^{+}}} \int_{0}^{\tau_{a+1 / n}^{+}} e^{-q t} d t, \tau_{a+1 / n}^{+}<\tau^{d}\right] \\
& +\mathbb{E}_{a}\left[r^{N_{\tau^{d}}} \int_{0}^{\tau^{d}} e^{-q t} d L_{t}^{a}, \tau^{d}<\tau_{a+1 / n}^{+}\right]
\end{aligned}
$$

since $L_{t}^{a}=\bar{X}_{t}-a$ under $\mathbb{P}_{a}$ can increase only by $1 / n$ each time the regulated process is above $a$ up to time $\tau_{a+1 / n}^{+}$. Moreover, since $r \leqslant 1$ we have

$$
\mathbb{E}_{a}\left[r^{N_{\tau^{d}}} \int_{0}^{\tau^{d}} e^{-q t} d L_{t}^{a}, \tau^{d}<\tau_{a+1 / n}^{+}\right] \leqslant \frac{1}{n} \mathbb{P}_{a}\left(\tau^{d}<\tau_{a+1 / n}^{+}\right)
$$

Note that we have $\lim _{n \rightarrow \infty} \mathbb{E}_{a}\left[r^{N_{\tau_{a+1 / n}^{+}}} \int_{0}^{\tau_{a+1 / n}^{+}} e^{-q t} d t, \tau_{a+1 / n}^{+}<\tau^{d}\right]=0$ and $\lim _{n \rightarrow \infty} \mathbb{P}_{a}\left(\tau^{d}<\tau_{a+1 / n}^{+}\right)=0$. All details of the above estimation can also be found in [27, proof of Proposition 1] where $T_{n}^{\prime}=\min \left\{\tau_{a+1 / n}^{+}, \tau^{0}\right\}$ should be changed to $T_{n}^{\prime}=\min \left\{\tau_{a+1 / n}^{+}, \tau_{0}^{d}\right\}$. Taking $b>a+1 / n$ in the definition of $g_{d}(x)$, from (5.1) we derive

$$
v^{a, d}(a)=\frac{g^{d}(a)}{g^{d}(a+1 / n)}\left(v^{a, d}(a)+\frac{1}{n}\right)+\mathrm{o}\left(\frac{1}{n}\right) .
$$

Hence

$$
v^{a, d}(a)=\frac{g^{d}(a)}{g^{d, \prime}(a)}=\frac{1}{\left(h^{d}\right)^{\prime}(a)} .
$$

The assertion of the theorem follows now from 3.8 and 5.3 . 
Proof of Lemma 3.7 For $r \in(0,1]$ and $q>0$ we define the joint Laplace transform of $N_{\tau_{y}^{+}}$and $\tau_{y}^{+}$:

$$
\phi(y):=\mathbb{E}\left[r^{N_{\tau_{y}^{+}}} e^{-q \tau_{y}^{+}} \mathbb{I}\left(\tau_{y}^{+}<\infty\right)\right]=\int_{0}^{\infty} e^{-q t} \sum_{k=0}^{\infty} r^{k} v_{y}(k, t) d t .
$$

First we assume that $\sigma>0$. Similarly to [19, Thm. 2] one can show that $\phi$ is twice differentiable and in contrast to the proof of Lemma 3.1 we do not have here any additional term, since there is no Parisian delay. Moreover, $\phi$ solves the integrodifferential equation

$$
\frac{\sigma^{2}}{2} \phi^{\prime \prime}(y)-c \phi^{\prime}(y)-(\lambda+\delta) \phi(y)+\lambda r \int_{0}^{\infty} \phi(y+x) f(x) d x=0 .
$$

Clearly, we have two boundary conditions:

$$
\begin{aligned}
& \phi(0)=1, \\
& \lim _{y \rightarrow \infty} \phi(y)=0 .
\end{aligned}
$$

Since the solution of 5.5 with the boundary conditions 5.6 and 5.7 is unique, we will check that $\phi(y)$ is of the form

$$
\phi(y)=e^{-b_{0} y}
$$

for some $b_{0}$. Note that the real part of $b_{0}$ must be positive, because otherwise it would be a contradiction to $\lim _{y \rightarrow \infty} \phi(y)=0$. Let $\rho$ be the unique solution to the Lundberg fundamental equation (3.24). Observe that

$$
\phi(y)=e^{-\rho y} .
$$

Taking the inverse Laplace transform with respect of $q$ it follows from (5.4) that

$$
\begin{aligned}
\sum_{k=0}^{\infty} r^{k} v_{y}(k, t) & =\mathcal{L}_{q}^{-1}\left(e^{-\rho y}\right) \\
& =\sum_{k=0}^{\infty} r^{k} \frac{\lambda^{k}}{k !} y t^{k-1} e^{-\lambda t} \int_{-\infty}^{\infty} \frac{1}{\sqrt{2 \pi t}} e^{-\frac{x^{2}}{2 t}} f^{k *}(c t+\sigma x-y) d x
\end{aligned}
$$

The details can be found in Czarna et al. [6]. The case of $\sigma=0$ could be proved in a similar way.

\section{REFERENCES}

[1] S. Asmussen and M. Taksar, Controlled diffusion models for optimal dividend pay-out, Insurance Math. Econom. 20 (1997), 1-15. 
[2] F. Avram, Z. Palmowski, and M. R. Pistorius, On the optimal dividend problem for a spectrally negative Lévy process, Ann. Appl. Probab. 17 (2007), 156-180.

[3] F. Avram, Z. Palmowski, and M. R. Pistorius, On Gerber-Shiu functions and optimal dividend distribution for a Lévy risk process in the presence of a penalty function, Ann. Appl. Probab. 25 (2015), 1868-1935.

[4] P. Azcue and N. Muler, Optimal reinsurance and dividend distribution policies in the CramérLundberg model, Math. Finance 15 (2005), 261-308.

[5] M. Chesney, M. Jeanblanc-Picqué, and M. Yor, Brownian excursions and Parisian barrier options, Adv. Appl. Probab. 29 (1997), 165-184.

[6] I. Czarna, Y. Li, Z. Palmowski, and C. Zhao, The joint distribution of the Parisian ruin time and the number of claims until Parisian ruin in the classical risk model, J. Comput. Appl. Math. 313 (2017), 499-514.

[7] I. Czarna and Z. Palmowski, Dividend problem with Parisian delay for a spectrally negative Lévy risk process, J. Optimization Theory Appl. 161 (2010), 239-256.

[8] A. Dassios and S. Wu, Parisian ruin with exponential claims, manuscript, 2008.

[9] B. de Finetti, Su un'impostazione alternativa della teoria collettiva del rischio, in: Transactions of the XVth International Congress of Actuaries, New York, 1957, 433-443.

[10] D. C. M. Dickson and C. Hipp, On the time to ruin for Erlang(2) risk processes, Insurance Math. Econom. 29 (2001), 333-344.

[11] H. U. Gerber, The dilemma between dividends and safety and a generalization of the LundbergCramér formulas, Scand. Actuarial J. 1974, 46-57.

[12] H. U. Gerber and E. S. W. Shiu, The joint distribution of the time of ruin, the surplus immediately before ruin, and the deficit at ruin, Insurance Math. Econom. 21 (1997), 129-137.

[13] H. U. Gerber and E. S. W. Shiu, Optimal dividends: analysis with Brownian motion, North Amer. Actuarial J. 8 (2004), 1-20.

[14] M. Jeanblanc-Picqué and A. N. Shiryaev, Optimization of the flow of dividends, Russian Math. Surveys 50 (1995), 257-277.

[15] A. Kuznetsov, A. E. Kyprianou, and V. Rivero, The theory of scale functions for spectrally negative Lévy processes, in: Lévy Matters II, Lecture Notes in Math. 2061, Springer, 2012, 97-186.

[16] A. E. Kyprianou, Fluctuations of Lévy Processes with Applications, Springer, 2014.

[17] A. Kyprianou and Z. Palmowski, A martingale review of some fluctuation theory for spectrally negative Lévy processes, in: Séminaire de Probabilités XXXVIII, Lecture Notes in Math. 1857, Springer, 2005, 16-29.

[18] A. E. Kyprianou and Z. Palmowski, Distributional study of de Finetti's dividend problem for a general Lévy insurance risk process, J. Appl. Probab. 44 (2007), 428-443.

[19] S. Li, The distribution of the dividend payments in the compound Poisson risk model perturbed by diffusion, Scand. Actuarial J. 2006, no. 2, 73-85.

[20] S. Li, The time of recovery and the maximum severity of ruin in a Sparre Andersen model, North Amer. Actuarial J. 12 (2008), 413-425.

[21] S. Li and J. Garrido, On ruin for the Erlang(n) risk process, Insurance Math. Econom. 34 (2004), 391-408.

[22] R. L. Loeffen, On optimality of the barrier strategy in de Finetti's dividend problem for spectrally negative Lévy processes, Ann. Appl. Probab. 18 (2008), 1669-1680.

[23] R. L. Loeffen, An optimal dividends problem with transaction costs for spectrally negative Lévy processes, Insurance Math. Econom. 45 (2009), 41-48.

[24] R. L. Loeffen, I. Czarna, and Z. Palmowski, Parisian ruin probability for spectrally negative Lévy processes, Bernoulli 19 (2011), 599-609.

[25] R. L. Loeffen and J. F. Renaud, De Finetti's optimal dividends problem with an affine penalty function at ruin, Insurance Math. Econom. 46 (2010), 98-108.

[26] Z. Palmowski and T. Rolski, A technique for exponential change of measure for Markov processes, Bernoulli 8 (2002), 767-785. 
[27] J. F. Renaud and X. Zhou, Distribution of the present value of dividend payments in a Lévy risk model, J. Appl. Probab. 44 (2007), 420-427.

[28] K. Sato, Lévy Processes and Infinitely Divisible Distributions, Cambridge Univ. Press, Cambridge, 1999.

[29] H. Schmidli, Optimisation in non-life insurance, Stochastic Models 22 (2006), 689-722.

Irmina Czarna, Zbigniew Palmowski

Faculty of Pure and Applied Mathematics

Wrocław University of Science and Technology

Wybrzeże Wyspiańskiego 27

50-370 Wrocław, Poland

E-mail: irmina.czarna@pwr.edu.pl zbigniew.palmowski@pwr.edu.pl

Chunming Zhao

Jiaotong University

Sichuan, 611756, P.R. China

E-mail:cmzhao@swjtu.cn
Yanhong Li

Sichuan University

Sichuan, 610065, P.R. China

E-mail:yanhongli@scu.edu.cn

Received 28.2.2018;

revised version 24.9.2018 
